\title{
Combined Excimer Laser and Topical Tacrolimus for the Treatment of Vitiligo: A Pilot Study
}

\author{
Adam Z. Kawalek, BA, ${ }^{*}$ James M. Spencer, MD, MS, ${ }^{*}$ and Robert G. Phelps, MD ${ }^{\dagger}$ \\ *Department of Dermatology and Department of Pathology, Division of Dermatologic Surgery, Mount Sinai School of \\ Medicine, New York, New York
}

BACKGROUND. Vitiligo is an acquired skin disorder that is characterized by well-defined, often symmetric white patches. Although current therapeutic modalities are directed toward increasing melanocyte melanin production, few treatment modalities address the immunologic nature of the disease.

OBJECTIVE. To determine whether excimer laser, a known therapeutic modality, in combination with tacrolimus, a topical immunomodulator, accelerate response time and/or improve the degree of response in patients with this disorder.

METHODS. Eight subjects diagnosed with vitiligo were recruited to participate in this institutional review board-approved double-blind, placebo-controlled study. Twenty-four symmetric vitiliginous patches (elbows, knees) from eight subjects received excimer laser treatment three times per week for 24 treatments or 10 weeks. Additionally, topical tacrolimus $0.1 \%$ ointment (Protopic) and placebo (Aquaphor) were applied to randomized patches (left or right) twice daily throughout the length of the trial. Vitiliginous patches were monitored with photographs at baseline, every 2 weeks, and 6 months after treatment. Biopsies were performed on subjects with significant results.

RESULTS. Twenty vitiliginous patches from six subjects qualified for evaluation. Fifty percent of patches treated with combination excimer laser and tacrolimus achieved a successful response ( $75 \%$ repigmentation) compared with $20 \%$ for the placebo group. Subjects who responded successfully repigmented faster $(\mathbf{1 9 \%})$ with combination therapy compared with excimer laser alone. Additionally, three subjects experienced transient hyperpigmentation in lesions treated with combination therapy.

CONCLUSION. Combining topical immunomodulators with known phototherapeutic modalities may represent a key advancement in the treatment of disease.
VITILIGO IS an acquired cutaneous depigmentation disorder affecting approximately $1 \%$ to $2 \%$ of the world population with no predilection for age, gender, or racial background. Although not life threatening, vitiligo is a disfiguring disorder and can have profound psychologic consequences. ${ }^{1}$ The basic pathogenesis of vitiligo remains unknown, although recent studies suggest genetic predisposition, relationship to other autoimmune disorders, biochemical and neurohormonal imbalance, and environmental toxins/stressors. ${ }^{2-4}$

Present therapies require many months to years of treatment and sometimes result in disappointing outcomes. ${ }^{5,6}$ Common therapeutic options include phototherapy with psoralen plus ultraviolet A (PUVA) and broadband or narrow-band ultraviolet $B$ (UVB) phototherapy. An analysis of common therapeutic agents used for the treatment of vitiligo is represented in Figure 1. ${ }^{7}$

Topical and systemic steroids, autologous melanocyte transplantation, and "home remedies," including

Address correspondence and reprint requests to: Adam Z. Kawalek, 322 West 72nd Street \#110, New York, NY 10023. placental extract, have all been met with mediocre success. Recently, clinical research has focused on the effects of topical immunomodulators (TIMs), pharmacologic agents that suppress or modulate dysregulated aspects of the immune system. ${ }^{8,9}$ Although the exact modulatory mechanism is unknown, ${ }^{10}$ patients with vitiligo often present with abnormalities of both humoral and cell-mediated immunity. ${ }^{11}$

Thus far, phototherapy has remained the most efficacious treatment option for people with vitiligo. ${ }^{12}$ With the recent advent of TIMs for the treatment of dermatologic conditions, we reasoned that combination phototherapy and TIM might act synergistically through activation of pathways influencing the process of melanocyte mitogenesis, melanocyte migration, and melanogenesis, ${ }^{13}$ thereby increasing the quality of repigmentation and/or hasten response times in patients with this disorder.

\section{Methods}

Eight subjects with multiple discrete chronic stable patches of vitiligo were enrolled in this pilot study. 


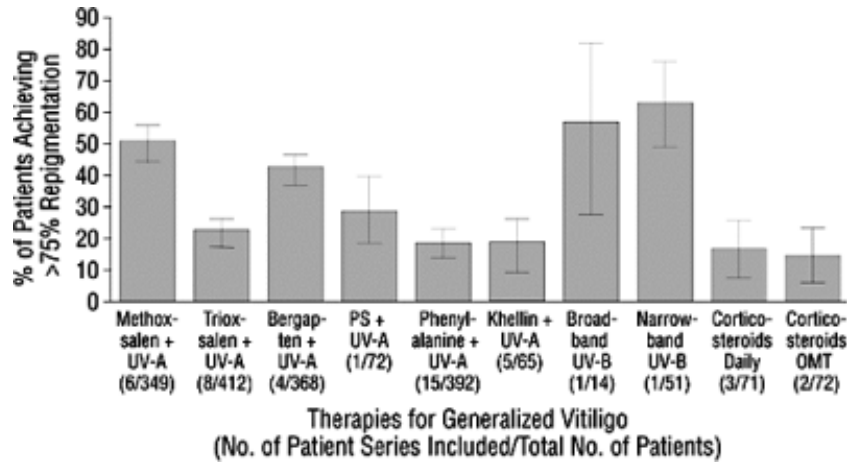

Figure 1. Effectiveness of therapies for generalized vitiligo. Analysis was based on patient series (sample size-weighted averages and 95\% confidence intervals). PS indicates unsubstituted psoralen; OMT, oral minipulse therapy.

Table 1. Basic Subject Data

\begin{tabular}{lc}
\hline Age & $(\#)$ \\
Mean & 38 \\
Range & $31-51$ \\
Sex & \\
Male & 3 \\
Female & 5 \\
Duration of disease & \\
$<1$ years & 1 \\
1-5 years & 1 \\
$>5$ years & 6 \\
Previous treatment & \\
PUVA* & 2 \\
Topical steroids & 3 \\
Systemic steroids & 1 \\
TIM & \\
Homeopathic & 2 \\
Family history & 3 \\
Vitiligo & \\
Thyroid disorder & 4 \\
Lupus & 1 \\
\hline
\end{tabular}

*PUVA = UVA and psoralen; ${ }^{\dagger}$ Aldara, Pimecrolimus.

Approval was obtained from the institutional review board at Mount Sinai School of Medicine, and written consent was obtained from all patients. Before initiating therapy, subjects were clinically evaluated, and past medical history, including the duration and progression of the disease, sites of vitiliginous lesions, and extent of cutaneous involvement, was obtained. Chronicity was defined as cutaneous depigmentation present for greater than 1 year. Subjects with rapidly progressing disease, evidence of spontaneous repigmentation, or treatment for their vitiligo within the last 6 months were excluded from the study. Additionally, only subjects with symmetric vitiliginous patches (left/right knee) were included in the study (Table 1).
Subjects with multiple lesions who had at least two symmetric patches were instructed to apply tacrolimus $0.1 \%$ (Protopic; Fujisawa) to one randomized symmetric patch and placebo cream (Aquaphor) to the other symmetric patch twice daily. Nonsymmetric patches were used as ultimate control subjects and received no therapy. The study was double blind. Test areas of vitiligo were treated with a $308-\mathrm{nm}$ xenonchloride excimer laser (Xtrac 308-nm laser; Photomedex, Radnor, PA). A 120-ns, 20-Hz pulse was used with a $10 \times 10$-mm spot size and a power output of $60 \mathrm{~mW}$ of monochromatic laser light. Lesions were evaluated and treated up to three times per week for a maximum of 24 treatments or 10 weeks, whichever came first. Exposure time was initiated at 2 seconds and increased by 2 seconds at every other visit unless erythema developed. In that case, the dose duration was held constant or reduced by 2 seconds until the erythema subsided. Treatment was withheld if sunburn or slight blistering developed and held until resolution, at which time treatment was resumed.

Treated areas were evaluated for repigmentation and erythema on separate point scales. Repigmentation was recorded as $0=$ no response, $1=$ minimal $(25 \%), 2=$ moderate $(25 \%$ to $75 \%), 3=$ marked $(75 \%)$, or $4=$ complete $(100 \%)$, depending on the extent of repigmentation for any specific lesion. A score of 3 or higher $(75 \%)$ was considered a successful treatment response. For the purpose of this study, partial repigmentation was defined as a repigmentation score of 1 or higher. Erythema was similarly graded as 0 (none), 1 (mild), 2 (moderate), or 3 (severe). Patients' Fitzpatrick skin type was noted before the start of treatment, and photographs were taken before treatment and every 2 weeks and at 6 months after treatment. Treatment was discontinued if subjects could not adhere to the treatment regimen.

\section{Results}

Table 2 summarizes the clinical features and degree of repigmentation in subjects. Twenty patches of vitiligo from six of eight subjects received enough treatment to qualify for evaluation. Two subjects could not adhere to the treatment regimen and were therefore dropped from the study. Eight of the 20 patches were located proximally (face and trunk), and 12 of the 20 were located on the extremities (wrists, knees, and elbows). Eight of 8 proximal patches $(100 \%)$ showed some degree of repigmentation compared with 10 of 12 patches on the extremities $(83 \%)$. As expected, subjects with the greatest repigmentation were Fitzpatrick skin types III-V. All six subjects $(100 \%)$ experienced some degree of repigmentation (Figures 2 and 3). 


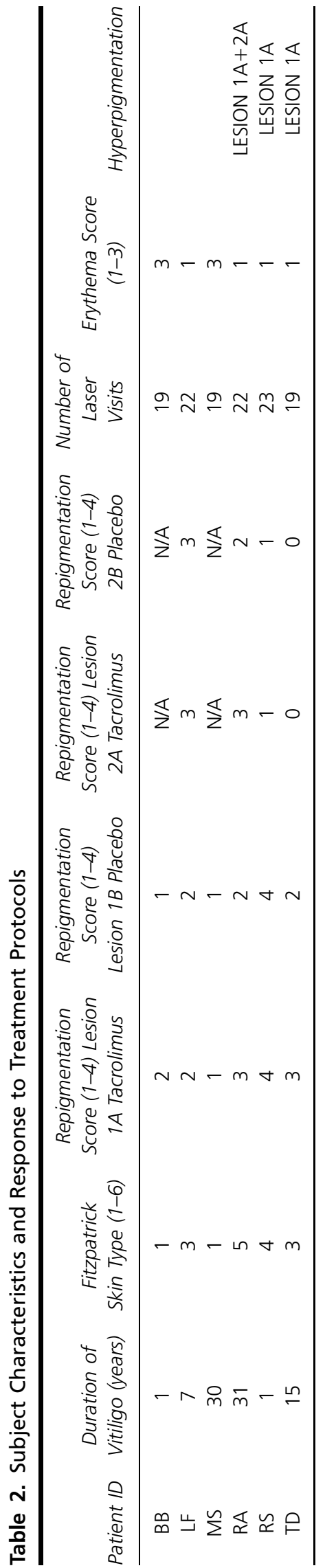

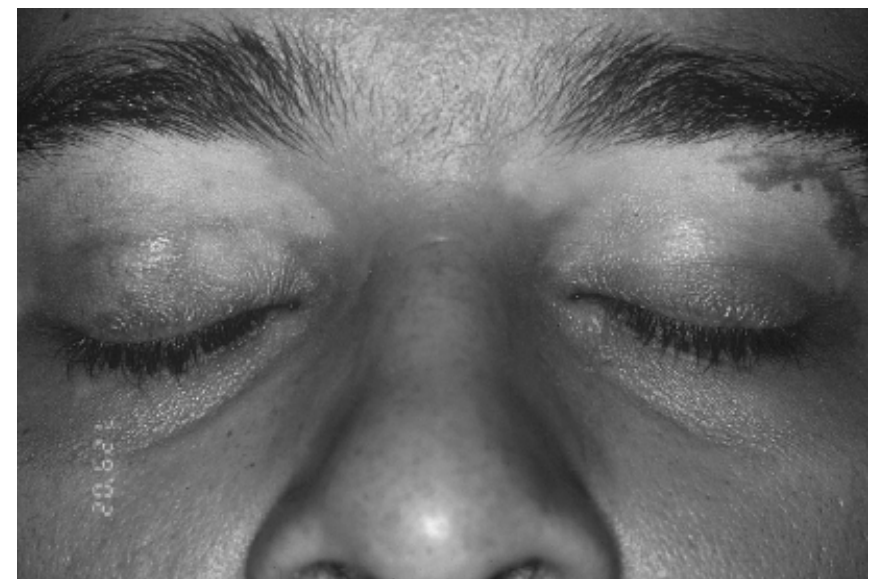

Figure 2. Subject R.S. before treatment.

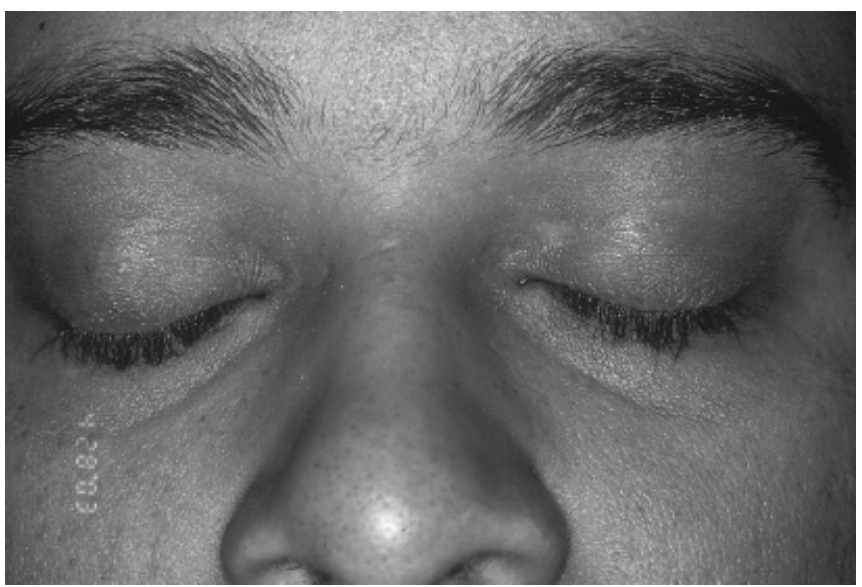

Figure 3. Subject R.S. 9 months after treatment.

Fifty percent of patches receiving combination excimer laser and tacrolimus achieved a successful response ( $75 \%$ repigmentation) compared with $20 \%$ for the placebo group, suggesting a significantly greater degree of repigmentation among patches treated with combination therapy compared with excimer laser alone (Table 3). Additionally, patches treated with excimer laser required an average of 16 treatments (53 days) to reach their respective end points. Comparatively, only 13 treatments (43 days) were required for lesions treated with combination therapy, suggesting a faster $(19 \%)$ rate of repigmentation among patches treated with combination excimer laser and topical tacrolimus (Table 4). In three subjects, patches treated with combination therapy remained one repigmentation score value higher than placebo patches (Table 2).

Three of six patients who completed the study $(50 \%)$ developed moderate lesional and perilesional hyperpigmentation on patches treated with combination 
Table 3. Number and Percentage of Patches Responding to Both Treatment Protocols

\begin{tabular}{lrr}
\hline Repigmentation Score & $\begin{array}{c}\text { \% Patches Responding to Excimer Laser } \\
\text { (placebo)/Total in Group }\end{array}$ & $\begin{array}{c}\text { \% Patches Responding to Combination } \\
\text { Therapy/Total in Group }\end{array}$ \\
\hline 0 & $10 \%(1 / 10)$ & $10 \%(1 / 10)$ \\
1 & $30 \%(3 / 10)$ & $20 \%(2 / 10)$ \\
2 & $40 \%(4 / 10)$ & $20 \%(2 / 10)$ \\
3 & $10 \%(1 / 10)$ & $40 \%(4 / 10)$ \\
4 & $10 \%(1 / 10)$ & $10 \%(1 / 10)$ \\
Successful response $3+4$ & $20 \%(2 / 10)$ & $50 \%(5 / 10)$ \\
\hline
\end{tabular}

All subjects had untreated control patches, none of which showed any repigmentation.

Table 4. Response Rates and Rate of Repigmentation Among All Patches Tested $(n=20)$

\begin{tabular}{lcr}
\hline & Tacrolimus 0.1\%+Excimer Laser & Excimer Laser Alone \\
\hline $\begin{array}{l}\text { Successful response (75\%) } \\
\text { Rate of repigmentation }\end{array}$ & $50 \%$ & $20 \%$ \\
\hline
\end{tabular}

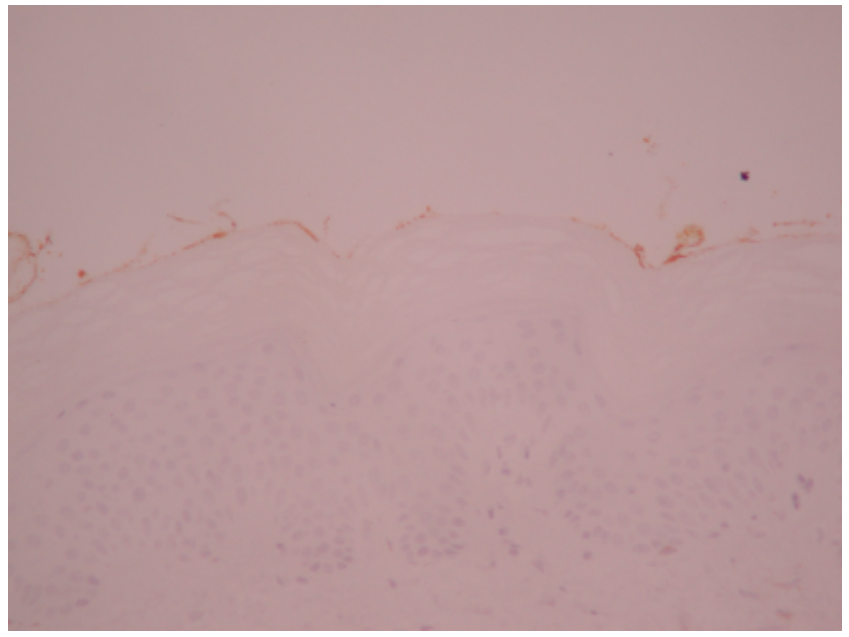

Figure 4. Melan A stain, magnification $\times 10$.

therapy. No control patches developed any degree of hyperpigmentation. For the purpose of this study, hyperpigmentation was defined as a color noticeably darker than the subject's normal skin tone. Additionally, all three were Fitzpatrick skin types III-V compared with I-III for the group that did not hyperpigment. The hyperpigmentation subsided in all three subjects within 3 weeks (18 days average) of completing the trial.

A 3-mm punch biopsy was taken from subject R.A. on both left- and right-treated knee patches in order to contrast the degree of repigmentation/ hyperpigmentation (Figures 4 and 5). The density and apparent activity of melanocytes in the biopsy lesion treated with combination therapy is evident. Note the greater number of melan A-positive cells (Figure 6) as

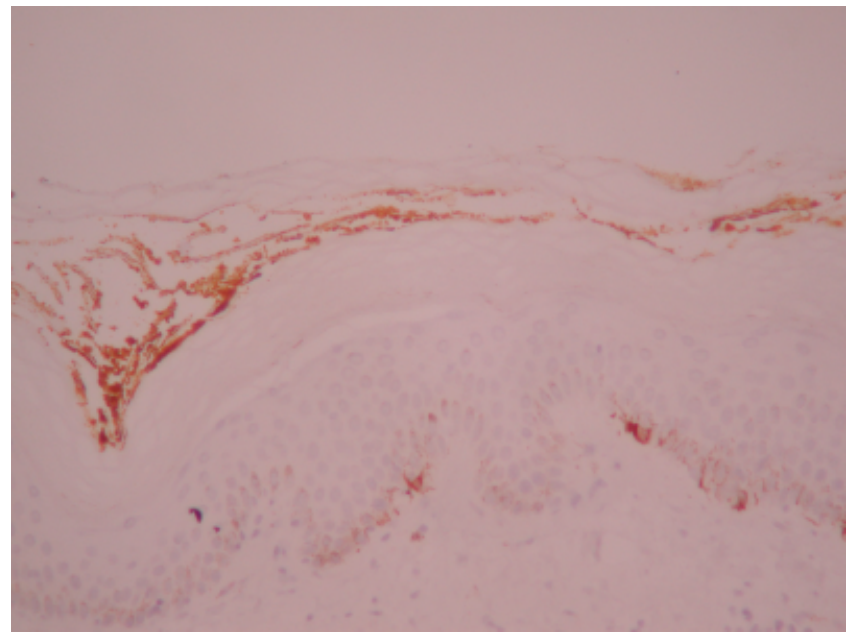

Figure 5. Melan A stain, magnification $\times 20$.

well as the increased number of langerhan cells overall (Figure 7).

Two patients dropped out of the study after six and eight treatments, respectively. Adherence to timeconsuming office visits was most likely responsible. Among those two, one subject had slight repigmentation (score of 1), and the other did not respond. There were no serious adverse events during the trial period. Mild to moderate erythema was observed in all 20 patches. Four patches received an erythema score of 3 (Table 2). One patch blistered slightly, and laser treatment was discontinued until symptoms subsided. Side effects for both the placebo $(30 \%)$ and topical tacrolimus $0.1 \%(80 \%)$ included tingling sensation, burning, and erythema. These effects quickly subsided after several days of treatment in all eight subjects. 


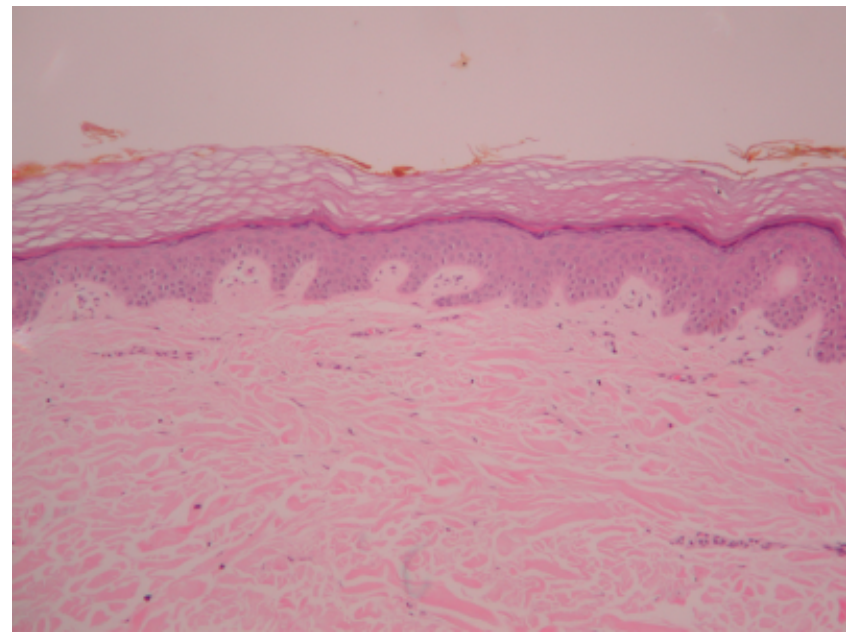

Figure 6. Hematoxylin and eosin stain, magnification $\times 10$.

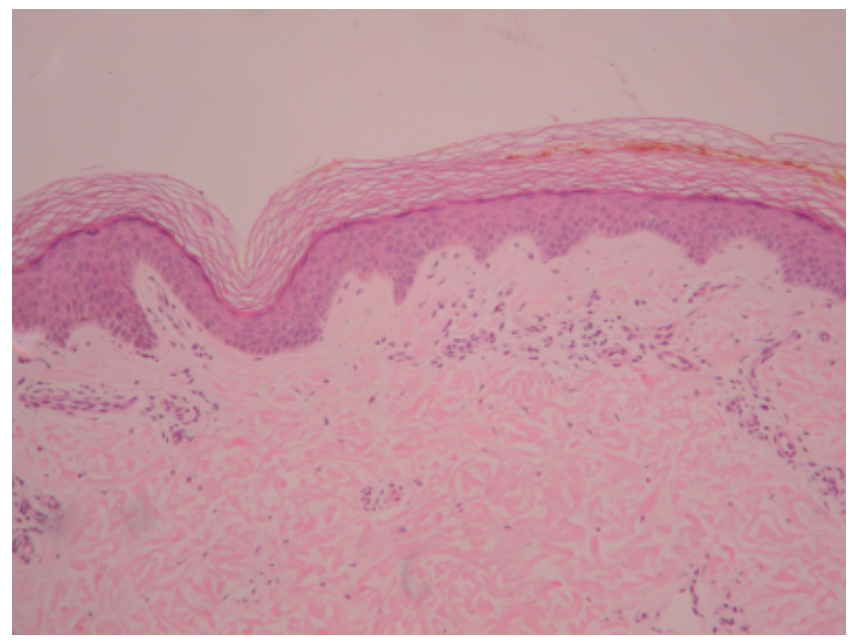

Figure 7. Hematoxylin and eosin stain, magnification $\times 10$.

\section{Discussion}

Current treatment modalities for vitiligo suppress the immune response and/or stimulate the proliferation of melanocytes with ultraviolet radiation. ${ }^{14}$ Unfortunately, current therapeutic options are limited by inconsistent and incomplete responses, relapses, and inconvenience of treatments. Phototherapeutic options, for example, are generally limited by the requirement of long-term treatment on a biweekly or triweekly basis over many months to years. Response rates for the standard modalities, including systemic and topical PUVA and broadband and narrowband UVB radiation, are highly variable, averaging approximately $51 \%$ for PUVA, $57 \%$ for UVB, and $63 \%$ for narrowband UVB. Although narrowband UVB radiation has been shown to be most efficacious with an optimal side-effect profile, reported treatment series require 6 to 12 months of treatment before optimal results are seen. ${ }^{15}$

Numerous observations support abnormalities in humoral and cellular immunity in patients with vitiligo. ${ }^{16,17}$ Because these abnormalities likely play a role in the induction of vitiligo, the use of immunosuppressive agents has been increasingly investigated for the treatment of this disease. Tacrolimus, a TIM, modulates the immune system by inhibiting $\mathrm{T}$-cell activation via downregulation of the transcription genes encoding proinflammatory cytokines, namely interleukin (IL)-2, IL-3, IL-4, IL-5; interferon- $\gamma$; tumor necrosis factor- $\alpha$; and granulocyte-macrophage colony-stimulating factor. ${ }^{18}$ Tacrolimus $0.1 \%$ is Food and Drug Administration approved for atopic dermatitis and is used off label for several other dermatologic conditions, including psoriasis. Although the mechanism of action of tacrolimus has been elucidated, data describing how tacrolimus modulates the cutaneous milieu remain unknown. ${ }^{19}$

Although the idea of combination therapy is not a new one, this is the first study to date that addresses the use of TIMs with laser therapy as a method of improving response rates in persons with vitiligo. In our series of subjects, we evaluated the efficacy of twice-daily double-blind placebo-controlled topical tacrolimus $0.1 \%$ in eight patients with generalized vitiligo involving less than $20 \%$ body surface area. Subjects were treated with 308-nm narrowband UVB excimer laser up to three times per week for a total of 10 weeks or 24 visits. Various degrees of repigmentation were achieved in all six subjects. Patches of vitiligo that were located proximally (face and trunk) responded to treatment $(100 \%)$ more than distal patches $(83 \%)$, reinforcing previous data that proximal vitiligo responds better to treatment. ${ }^{20}$ Of the 20 vitiligo patches from the six subjects who completed the trial, $50 \%$ of patches treated with combination therapy achieved $75 \%$ repigmentation and therefore successfully responded. Only $20 \%$ of patches treated with excimer laser and placebo achieved a successful response. The patches treated with combination therapy also repigmented at a significantly faster rate, suggesting that topical tacrolimus hastens response time in patients with this disorder. The longevity of repigmentation was not addressed in this study. However, from experience with PUVA and narrowband UVB, repigmentation is permanent. Of special interest, three of the six subjects experienced hyperpigmentation on patches treated with combination therapy versus excimer laser alone. Hyperpigmented patches regressed to normal skin tone within 3 weeks in all three subjects, suggesting some degree of normal melanocyte function in these subjects. 
Minimal burning and stinging of the skin occurred in most subjects $(80 \%)$ at the initiation of treatment with tacrolimus ointment. Tacrolimus ointment was also well tolerated in the axillary and periocular areas. All subjects were previously refractory to treatment, and six of eight subjects had vitiligo for over 5 years, reinforcing previous reports that there is no relationship between duration of disease, progression, and therapeutic result. ${ }^{21}$

Conventional phototherapy delivers UV radiation to affected and unaffected skin over many months of treatment. The possibility of increased risk of skin cancer after phototherapy has been repeatedly raised. Targeted phototherapy with the 308-nm excimer laser delivers UV radiation to only the affected area. Uninvolved skin is not exposed. This limits total skin exposure to UV radiation and therefore may decrease the risk of skin cancer. ${ }^{5}$ Furthermore, the excimer laser provides a faster therapeutic effect than conventional phototherapy, and fewer total treatments are needed, limiting cumulative exposure to UV radiation.

Recent studies reporting on the efficacy of tacrolimus for the treatment of vitiligo have shown promising results. A correlation between the effectiveness of tacrolimus and exposure to sunlight was observed. ${ }^{22}$ Although excimer laser is a coherent and narrow wavelength, our study supports the conclusion that tacrolimus exerts a greater effect in the presence of low-frequency, high-energy ultraviolet light. The ease of topical self-administration with minimal side effects makes this novel immunomodulatory agent a promising addition to current treatment strategies. Future studies that explore other wavelengths, including infrared, and expand trials to a greater number of subjects especially skin types III-VI are needed.

The results of this study suggest that combination tacrolimus and excimer laser enhances response time and repigmentation rates in subjects with vitiligoespecially those with Fitzpatrick skin types III-IV. Because conventional phototherapy is time consuming, agents that enhance repigmentation and hasten therapeutic response times are welcome. Larger prospective studies demonstrating statistical significance of this novel therapeutic intervention are recommended in order to delineate further the efficacy of targeted phototherapy combined with TIMs.

Acknowledgment The authors thank Daniel Carrasco, MD, and Suhail Hadi, MD, for their support and assistance in completing this trial.

\section{References}

1. Hautmann G, Panconesi E. Vitiligo: a psychologically influenced and influencing disease. Clin Dermatol 1997;15:879-90.

2. Castenet J, Ortonne JP. Pathophysiology of vitiligo. Clin Dermatol 1997; 15:845-51.

3. Kemp EH, Waterman EA, Weetman AP. Autoimmune aspects of vitiligo. Autoimmunity 2001;34:65-77.

4. Ongenae K, Van Geel N, Naeyaert JM. Evidence for an autoimmune pathogenesis of vitiligo. Pigment Cell Res 2003;16: 90-100.

5. Spencer JM, Nossa R, Ajmeri J. Treatment of vitiligo with the 308nm excimer laser: a pilot study. J Am Acad Dermatol 2002;46: 727-31.

6. Grimes PE, Soriano T, Dytoc MT. Topical tacrolimus for repigmentation of vitiligo. J Am Acad Dermatol 2002;47:789-91.

7. Njoo MD, Spuls PI, Bos JD, Westerhof W, Bossuyt PM. Nonsurgical repigmentation therapies in vitiligo: meta-analysis of the literature. Arch Dermatol 1998;134:1532-40.

8. Grimes PE. Vitiligo: an overview of therapeutic approaches. Dermatol Clin 1993;11:325-7.

9. Zabawski EJ, Costner M, Cohen JB, Cockerell CJ. Tacrolimus: pharmacology and therapeutic uses in dermatology. Int J Dermatol 2000;39:721-7.

10. Thomson AW, Bonham CA, Zeevi A. Mode of action of tacrolimus (FK506): molecular and cellular mechanisms. Ther Drug Monit 1995;17:584-91.

11. Das PK, van den Wijngaard RM, Wankowicz-Kalinska A, Le Poole IC. A symbiotic concept of autoimmunity and tumour immunity: lessons from vitiligo. Trends Immunol 2001;22:130-6.

12. Njoo MD, Spuls PI, Bos JD, Westerhof W, Bossuyt PM. Nonsurgical repigmentation therapies in vitiligo. Arch Dermatol 1998;134:1532-40.

13. Castanedo-Cazares JP, Lepe V, Moncada B. Repigmentation of chronic vitiligo lesions by following tacrolimus plus ultraviolet-Bnarrow-band. Photodermatol Photoimmunol Photomed 2003;19: $35-6$.

14. Grimes PE. Diseases of hypopigmentation. In: Sams WM, Lynch PJ, eds. Principles and Practice of Dermatology, 2nd ed. New York: Churchill-Livingstone, 1996:873-85.

15. Njoo MD, Bos JD, Westerhof W. Treatment of generalized vitiligo in children with narrow-band (TL-01) UVB radiation therapy. J Am Acad Dermatol 2000;42:245-53.

16. Lang KS, Caroli CC, Muhm A, et al. HLA-A2 restricted, melanocyte-specific CD8 (+) $\mathrm{T}$ lymphocytes detected in vitiligo patients are related to disease activity and are predominantly directed against MelanA/MART1. J Invest Dermatol 2001;116: $891-7$.

17. Ogg GS, Rod DP, Romero P, Chen JL, Cerundolo V. High frequency of skin-homing melanocyte-specific cytotoxic $\mathrm{T}$ lymphocytes in autoimmune vitiligo. J Exp Med 1998;188:1203-8.

18. Lawrence I. Tacrolimus (FK 506) experience in dermatology. Dermatol Ther 1998;5:74-84.

19. Rico MJ, Lawrence I. Tacrolimus ointment for the treatment of atopic dermatitis: clinical and pharmacologic effects. Allergy Asthma Proc 2002;23:191-7.

20. Yalcin B, Sahin S, Bukulmez G, et al. Experience with calcipotriol as adjunctive treatment for vitiligo in patients who do not respond to PUVA alone: a preliminary study. J Am Acad Dermatol 2001; 44:634-7.

21. Acikel C, Ulkur E, Celikoz B. Carbon dioxide laser resurfacing and thin skin grafting in the treatment of "stable and recalcitrant" vitiligo. Plast Reconstr Surg 2003;111:1291-8.

22. Tanghetti EA. Tacrolimus ointment $0.1 \%$ produces repigmentation in patients with vitiligo: results of a prospective patient series. Cutis $2003 ; 71: 158-62$. 
This document is a scanned copy of a printed document. No warranty is given about the accuracy of the copy. Users should refer to the original published version of the material. 